\title{
SÉANCE DU 9 JUIN 1905
}

\author{
PRÉSIDENCE DE M. ED. BUREAU.
}

Il est donné lecture du procès-verbal de la précédente séance dont la rédaction est adoptée.

M. le Président a le regret d'annoncer à l'assemblée les décès de deux de nos Confrères.

M. Eug. Niel, décédé à Saint-Aubin-le-Vertueux (Eure), le 17 mai 1905, à l'âge de 69 ans, était admis dans notre Société en 1880. Il est l'auteur d'une Flore de l'Eure et de divers articles publiés dans le Bulletin ${ }^{1}$. Il collabora également aux Bulletins de la Société mycologique de France, de la Société des amis des sciences de Rouen, de la Société Linnéenne de Normandie.

M. Aug. Michel, décédé à Carrières-sous-Bois le 25 mai dernier, était entré dans notre Société en 1863 et fut proclamé membre perpétuel le 12 avril 1889 .

M. le Président a reçu notification que la subvention de 1000 fr. sera continuée cette année par le Ministère de l'Instruction publique. M. le Président dit qu'il écrira une lettre de remerciements à $\mathrm{M}$. le Ministre en lui affirmant que la Société se rendra de plus en plus digne de ses bienveillants encouragements par les nombreux services qu'elle compte rendre à la science.

La Société a reçu deux envois de plantes fraîches que se partagent les membres présents; l'un d'eux contenait les espèces suivantes récoltées par M. A. Reynier aux environs de Marseille : Nigella damascena, Alyssum maritimum, Silene brachypetala, Arenaria massiliensis, Astragalus sesameus, A. hamosus, Onobrychis rquidentata, Medicago disciformis, Trifolium stellatum, Hyoseris radicata, Phlomis fruticosa, Psilurus nardoides.

1. En 1882 , p. $115 ; 1883$, p. $196 ; 1888$, pp. 112 et 373 , et 1889 , p. 256 . T. L.II.

(SÉANCES) 24 
Le second envoi, récolté au Lautaret par M. Offner, était composé de plantes alpines : Ranunculus pyrenæus, Pulsatilla alpina, P.vernalis, Viola calcarata, Saxifraga retusa, S. oppositifolia, Gentiana verna, G. brachyphylla, G. Kochiana, Primula viscosa, P. farinosa, Douglasia Vitaliana, Androsace carnea, Pulmonaria azurea, Pedicularis comosa, Ajuga pyramidalis, Alnus viridis, Orchis sambucina, Gagea fistulosa.

M. de Rey-Pailhade a envoyé un Hypecoum glaucescens récolté en avril à Serignac-Valras (Hérault).

M. le Président présente 39 plantes alpines vivantes provenant des cultures de $\mathrm{M}$. Ph. de Vilmorin.

Allium cæruleum Pall. - Sibérie. Brodiæa coccinea A. Gray. - Californie.

- lactea S. Wats. - Californie.

Bruckenthalia spiculifolia Reichb. - Europe orientale.

Calceolaria plantaginea Smith. Magellan.

Campanula alliariæfolia Willd. Caucase.

- garganica Tenore var. hirsuta Hort. - Italie.

- Hostii var. alba Hort.

- Van-Houttei Hort.

Carmichælia australis R. Br. - Nouvelle-Zélande.

Convolvulus Cneorum L. - Europe mérid.

Dianthus spec.? (à déterminer).

Digitalis lutea L. - Europe.

Discaria longispina Miers. - Uruguay.

Erigeron glaucus Ker-Gawl (typica). - Am. sept.

Erpetion reniforme Sweet. - Australie.

Genista dalmatica Bartl. et Wendl. - Dalmatie.

Helianthemum lunulatum DC. Alpes.
Helianthemum vulgare Gærtn. var. roseum $D C$. - Europe.

- rosmarinifolium Lag. - Espagne.

Hieracium aurantiacum L. - Europe.

Hypericum polyphyllum Boiss. Cilicie.

Lactuca perennis L. var. alba Hort. - Europe.

Libertia formosa Graham. - Chili.

Lilium bulbiferum L. - Europe.

- Hansoni Leicht. - Japon.

- sutchuenense Franch. - Sutchuen.

Linum arboreum $L$. - Crète.

Mazus Pumilio R. Br. - Nouvelle-Zélande.

Mimulus floribundus Lindl. - Californie.

Phlox carolina L. - Am. sept.

Pratia angulata Hook. f. - NouvelleZélande.

Saxifraga cæsia L. - Alpes.

- cuscutæformis Lodd. - Japon.

Sisyrinchium convolutum Nocca. Am. trop.

- striatum Smith. - République Argentine.

Veronica prostrata $L$. - Europe. 


\section{$2 \mathrm{BHL}$ Biodiversity Heritage Library}

1905. "Séance Du 9 Juin 1905." Bulletin de la Société botanique de France 52, 369-370. https://doi.org/10.1080/00378941.1905.10829165.

View This Item Online: https://www.biodiversitylibrary.org/item/8674

DOI: https://doi.org/10.1080/00378941.1905.10829165

Permalink: https://www.biodiversitylibrary.org/partpdf/160519

\section{Holding Institution}

Missouri Botanical Garden, Peter H. Raven Library

\section{Sponsored by}

Missouri Botanical Garden

\section{Copyright \& Reuse}

Copyright Status: Public domain. The BHL considers that this work is no longer under copyright protection.

This document was created from content at the Biodiversity Heritage Library, the world's largest open access digital library for biodiversity literature and archives. Visit BHL at https://www.biodiversitylibrary.org. 